\title{
An Overview of Scientific-Based Knowledge on Sedentary Behaviour Among the Pediatric Population - A Conceptual Model Development
}

\section{Salomé AUBERT* ${ }^{*}$}

1 Student, University of Ottawa, Canada

*Auteur(e) correspondant | Corresponding author : saubeo82@uottawa.ca

Résumé :

(traduction)
La sédentarité a été établie comme un comportement nuisible qui affecte de multiples aspects de la santé des personnes de tous âges, y compris des enfants et des jeunes de o à 18 ans. Le présent rapport vise à (i) résumer la documentation qui porte sur la définition, les caractéristiques, le contexte, les déterminants et les conséquences de la sédentarité chez les enfants et les jeunes; (ii) proposer un modèle conceptuel qui résume les résultats; (iii) souligner les lacunes de la recherche présentée dans la documentation. La sédentarité est un comportement commun et complexe qui risque d'affecter la santé des enfants et des jeunes sur les plans physiologique et psychologique. Ses effets néfastes sur la santé varient selon la manière dont ils s'accumulent au cours d'une journée, par exemple, selon le nombre d'interruptions ou le contexte. La sédentarité infantile étant trop élevée dans la plupart des pays où elle a été évaluée, le développement de la recherche et l'élaboration de politiques qui ciblent la réduction de la sédentarité chez les enfants et les jeunes doivent figurer au sommet des priorités pour les organismes de santé publique partout dans le monde. Même s'il faut poursuivre la recherche sur l'identification des conséquences et des déterminants particuliers des différents types et modèles de sédentarité dans divers contextes, le modèle conceptuel de l'étude et l'interprétation de la sédentarité chez les enfants et les jeunes, donnent un aperçu du sujet et appuient l'élaboration de politiques et le développement de la recherche.

Comportement sédentaire, enfants, jeunes, santé de la population 


\begin{abstract}
:
Sedentary behaviour has been identified as a specifically deleterious personal practice on multiple levels of health of individuals at all ages, including the pediatric population (i.e., o to 18 years of age). The aims of this paper are to (i) summarize the literature concerning the definition, the characteristics, the context, the determinants, and the consequences of sedentary behaviour among children and youth; (ii) propose a conceptual model that summarizes these findings; and (iii) highlight research gaps in the literature. Sedentary behaviours are common and complex behaviours that can potentially affect the health of children and youth on the physiological and psychological levels. Those deleterious effects on health can vary depending on how they are accumulated throughout the day, for example with or without interruption, or in which context. While childhood engagement in sedentary behaviours is too high in the majority of countries where it has been assessed, developing research and policies that target the reduction of sedentary behaviours among children and youth must be ranked as a top priority for all public health organizations worldwide. Although further research is needed concerning the identification of the specific determinants and consequences of different types and patterns of sedentary behaviours in various contexts, the Conceptual Model for the Study and Understanding of Children and Youth's Sedentary Behaviour, gives an overview of the topic and supports the development of policy and further research.
\end{abstract}

Keywords:

Sedentary behaviour, children, youth, population health 


\section{Introduction}

The Public Health Agency of Canada (2013) defines population health as the health of a population as measured by health status indicators and influenced by social, economic and physical environments, personal health practices, individual capacity and coping skills, human biology, early childhood development, and health services. Sedentary behaviour has been identified as a specifically deleterious personal practice on multiple levels of health of individuals at all ages, including the pediatric population (Owen, Healy, Matthews, \& Dunstan, 2010; Tremblay, Colley, Saunders, Healy, \& Owen, 2010; Tremblay et al., 2011). The general objective of this paper is to present a pedagogic overview of the scientific knowledge concerning sedentary behaviour among children and youth to inform broad, evidence-based environmental and policy initiatives and to support the development of further research. To reach this goal, this paper will (i) review the literature to identify definitions, characteristics, contexts, determinants, and consequences of sedentary behaviour among children and youth; (ii) propose a conceptual model that summarizes these findings; and (iii) highlight research gaps in sedentary behaviour.

\section{Sedentary behaviours; definition and dis- tinction}

The study of the health impact of sedentary behaviour is an important emerging area of research. It has the potential to "suggest novel options for the prevention of noncommunicable disease and to suggest environmental innovations and new policies for preserving and enhancing population health" (Tremblay et. al., 2010). But to allow optimal development of sedentary behaviour related research, such as the study of its determinants and the creation of intervention for its reduction, clarity on the use of specific terms is needed.

Indeed, sedentary behaviour and physical inactivity are two distinct concepts that have been and are still confused in the literature. According to the Sedentary Behavior Research Network (2012), sedentary behaviour is "any waking behaviour characterized by an energy expenditure $\leq 1.5$ metabolic equivalents, while in a sitting or reclining posture." In contrast, the term "inactive" must be used to describe those who are performing insufficient amounts of moderate to vigorous physical activity (i.e., not meeting specified physical activity guidelines). Physical activity and sedentary behaviour can be seen as a continuum on the human movement spectrum (Gibbs, Hergenroeder, Katzmarzyk, Lee, \& Jakicic, 2014). In addition, being sedentary is not necessarily associated with being inactive; an active individual can meet physical activity guidelines while spending the vast majority of the day sitting (Saunders, Chaput, \& Tremblay, 2014) (Figure 1).

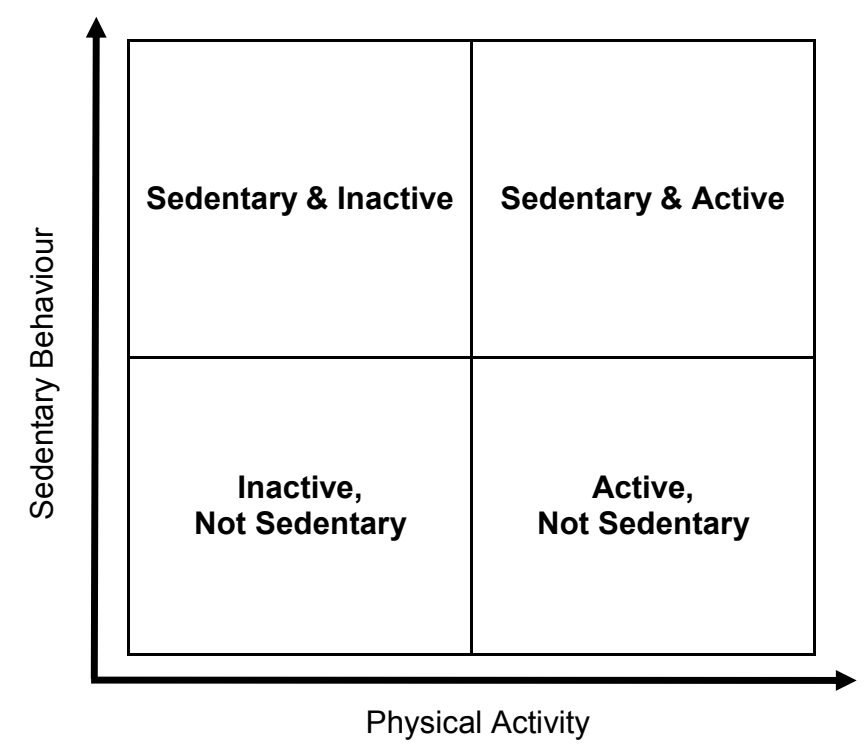

\section{Figure 1}

Sedentary behaviour and physical activity as distinct constructs. Reprinted from "Sedentary Behaviour as an Emerging Risk Factor for Cardiometabolic Diseases in Children and Youth," by T. J. Saunders, J.-P. Chaput, and M. S. Tremblay, 2014, Canadian Journal of Diabetes, 38 , p. 54. Copyright 2014 by Canadian Diabetes Association. Reprinted with permission.

\section{Sedentary behaviours; complexity of the phenomenon}

Sedentary behaviours are ubiquitous and encompass a range of activities. Sedentary behaviours, such as sitting on a sofa while watching TV or playing a board game with friends, can have very different characteristics and can occur in very different contexts, thus their determinants and consequences will not be the same. Recent evidence suggests that different types of sedentary behaviours have different impacts on health and wellbeing (Chastin, Schwarz, \& Skelton, 2013). For instance, some sedentary behaviours can have potential health-enhancing effects (rest and relaxation can be an essential need). In addition, research suggests that the consequences of sedentary behaviours on health depend greatly on their pattern (i.e., how they are accumulated throughout the day). For example, the same total amount of sedentary time would be associated with fewer negative health outcomes if it is accumulated with regular interruptions than if it is continuous (Healy et al., 2008). Understanding the sedentary behaviour setting the physical and social context where it occurs - is of particular importance to the study of its consequences on health (Owen et al., 2011). 
To characterize the sedentary behaviours, an open science project called Sedentary behaviour International Taxonomy project (SIT) was setup to develop a common taxonomy of sedentary behaviours through formal consensus, taking into account the opinion of experts and the general public. The project uses the Delphi method, a technique used for "the elicitation of opinions with the object of obtaining a group response from a panel of experts. It replaces direct confrontation and debate by a carefully planned, orderly program of sequential individual interrogations usually conducted by questionnaires" (Brown, 1968). The first round of this SIT project involved international experts who were asked to make statements about the taxonomy (its purpose and use), the domains, categories or facets that should be considered and included, and the structure/architecture to arrange and link these domains and facets (Chastin et al., 2013). The project aimed to facilitate systematic and standardized investigation and analysis to enable systematic and standardized reporting, to facilitate comparison, and to develop measurement tools of sedentary behaviours.

The obtained taxonomy comprises nine complementary facets (Figure 2) characterizing the purpose (why), the environment (where), the social context (with whom), the type or modality (what), associated behaviours (what else), when the behaviour takes place (when), the mental and functional states of sedentary individual (state), posture, and measurement and quantification issues (Chastin et al., 2013). Each of these facets has more precise sub-domains and categories that can be used to describe sedentary behaviours. For ex-

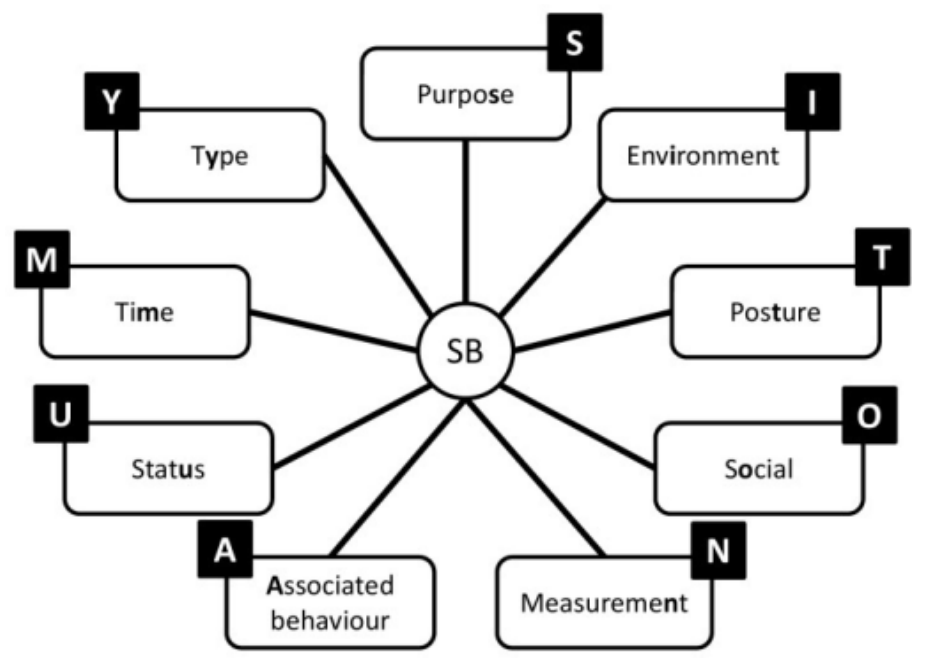

\section{Figure 2}

Taxonomy level one facets and coding labels. Reprinted from "Development of a Consensus Taxonomy of Sedentary Behaviors (SIT): Report of Delphi Round 1," by S. F. Chastin, U. Schwarz, and D. A. Skelton, 2013, PLoS One, 8, p. 7. Copyright 2013 by Chastin et al. Reprinted with permission. ample, the main facet of posture is composed of two subcategories: sitting and lying (Chastin et al., 2013).

Quantitatively, the following are used to characterize sedentary behaviours: frequency (number of bouts of certain duration); interruptions or breaks (period spent not sedentary between two bouts of sedentary behaviour); and time (total duration of sitting/lying while awake) (Tremblay et al., 2010). A definition of "sedentary bouts" and "sedentary breaks" has been recently proposed by Altenburg and Chinapaw (2015): sedentary bout is "a minimum period of uninterrupted sedentary time" and sedentary break is "a non-sedentary period in between two sedentary bouts." Research suggests that the consequences of sedentary behaviours on health depend greatly on how they are accumulated throughout the day. For example, the same total amount of sedentary time is associated with fewer negative health outcomes if it is broken up by regular interruptions instead of being continuous (Healy et al., 2008).

This growing body of research also confuses the use of sedentary behaviour-related concepts, thus, there is a need for adopting standardized terms to describe sedentary behaviours. An international team of over 80 researchers under the supervision of Dr. Mark Tremblay and the Sedentary Behaviour Research Network recently published the Terminology Consensus Project (Tremblay et al., 2017). This project proposed final consensus definitions for stationary behaviour, sedentary behaviour, standing, screen time, nonscreen-based sedentary time, sitting, reclining, lying, and sedentary behaviour pattern, including caveats and examples for all age groups and abilities, and a conceptual model integrating these terms.

\section{Consequences of sedentary behaviour in children and youth, a population health issue}

Increasing evidence suggests that sedentary behaviour, independent of physical activity level, is associated with negative outcomes at macroscopic and microscopic levels. Because the study of sedentary behaviour is a new area in health research, solid prospective findings among children and youth are still missing, but a review of the emergent literature is presented here.

Accumulating evidence suggests that the amount of time children and youth spend engaging in sedentary activities may be associated with increased cardiometabolic disease risk, independent of other factors such as physical activity level and abdominal obesity (Carson et al, 2016; Saunders et al., 2014). There is also an assumed association between prolonged sedentary behaviours and mental health indicators such as hyperactivity/inattention, internalizing problems, psychological well-being, and perceived quality of life 
among school-aged children and adolescents, but further investigation is required to confirm this relationship (Suchert, Hanewinkel, \& Isensee, 2015). A recent systematic review of sedentary behaviour and health indicators in school-aged children and youth (5 to 17 years old) found that higher durations of TV viewing and video game use were associated with unfavourable behavioural conduct, higher duration of screen time with lower fitness, and higher durations of screen time and computer use with lower self-esteem (Carson et al., 2016). Findings from numerous studies also suggest that sedentary screen-based behaviours (particularly television viewing) are likely to result in increased energy intake and positive energy balance in the pediatric population (Saunders et al., 2014). In addition, sedentary behaviours have been shown to coexist with other unhealthy behaviours such as higher energy intake from fat, sweet and salty snacks, and high-energy drinks (Biddle, Petrolini, \& Pearson, 2014; Salmon, Tremblay, Marshall, \& Hume, 2011), lower consumption of fruits and vegetables (Salmon et al., 2011), lower levels of physical activity (Biddle et al., 2014; Salmon et al., 2011), and inadequate sleep (Biddle et al., 2014). Other research has reported a significant positive association between media exposure (e.g., TV viewing, reading magazines) and the use of tobacco, drugs, or alcohol among youth (Salmon et al., 2011). Finally, according to a systematic review by Carson et al. (2015), increased screen time has detrimental associations with cognitive development outcomes in $38 \%$ of young children aged o to 5 years, while $6 \%$ report beneficial associations with cognitive developmental outcomes.

On a biological level, prolonged and uninterrupted sedentary behaviour has rapid and deleterious effects on insulin sensitivity, glucose tolerance, and triglyceride levels in adults, whereas interruptions in sedentary time benefit triglyceride and glucose metabolism (Saunders et al., 2014). To date, these findings still need to be replicated in the pediatric population. As for the pediatric population, a systematic review examining the relationship between sedentary behaviour and adiposity among school-aged children reported that 94 of 119 cross-sectional studies observed a positive association between sedentary behaviour and markers of adiposity, including higher serum cholesterol level, high systolic and diastolic blood pressure, and insulin resistance (Tremblay et al., 2011).

It is important to highlight that many studies on this topic have used "screen-time" (i.e., time spent in front of a TV or computer, assumed to be while sitting) as an indicator of sedentary behaviour instead of the total time spent being sedentary, in front of a screen or otherwise. It can lead to an underestimation of sedentary time and does not allow associations to be made about particular types of sedentary be- haviour. For example, Carson et al.(2015) found that reading or being read to is most consistently associated with positive cognitive development while the vast majority of evidence suggests that screen time has either no effect or a detrimental effect on cognitive development during early childhood (o to 5 years).

Finally, it is well recognized that behavioural patterns related to physiological risk factors (such as food choices, smoking, or physical activity) are fully established and resistant to change at the end of childhood and especially at the end of adolescence (Kelder, Perry, Klepp, \& Lytle, 1994). Thus, youth who exhibit high levels of sedentary behaviour are more likely to continue to engage in high levels of sedentary behaviours as adults.

\section{Situational analysis of children and youth's sedentary behaviours}

\subsection{Sedentary behaviour guideline for children and youth}

In response to the accumulation of research showing the deleterious effects of sedentary behaviour, insufficient physical activity, and/or insufficient/poor quality sleep in the pediatric population, the first Canadian 24-Hour Movement Guidelines for Children and Youth were published in 2016, integrating physical activity, sedentary behaviour, and sleep (Tremblay, Carson, \& Chaput, 2016a). Recommendations on sedentary behaviour state that:

For optimal health benefits, children and youth (aged 5-17 years) should achieve high levels of physical activity, low levels of sedentary behaviour, and sufficient sleep each day. A healthy 24 hours includes: [...] no more than 2 hours per day of recreational screen time; limited sitting for extended periods. (CSEP, 2016)

The same team is developing guidelines for younger children, between o to 4 years of age. In the first Canadian Sedentary Behaviour Guidelines for the Early Years that were published in 2012, it was recommended that:

For healthy growth and development, caregivers should minimize the time infants (aged $<1$ year), toddlers (aged 1-2 years) and preschoolers (aged 34 years) spend being sedentary during waking hours. This includes prolonged sitting or being restrained (e.g., stroller, high chair) for more than $1 \mathrm{~h}$ at a time. For those under 2 years, screen time (e.g., $\mathrm{TV}$, computer, electronic games) is not recommended. For children 2-4 years, screen time should be limited to under $1 \mathrm{~h}$ per day; less is better.

(Tremblay and al., 2012, p.375) 
With these guidelines come new indicators and new opportunities for surveillance. Adherence to these guidelines should be assessed at a population level internationally to evaluate: i) the need for and the efficacy of interventions, ii) the comparisons between countries and/or specific populations, and iii) the determinants and health consequences associated with sedentary behaviour.

\subsection{Prevalence of sedentary behaviour among children and youth in the world}

In order to promote a healthy and active lifestyle among children and youth, the Active Healthy Kids Canada Report Card has been released every year between 2005 and 2016. This Report Card is a harmonized process which uses common indicators of physical activity and a standardized grading framework from A ("succeeding with a large majority of children and youth, $\geq 80 \%$ ") to F ("succeeding with very few children and youth, $<20 \%$ ") to mark each of these indicators. This process has been replicated in low, middle, high, and very high-income countries - 14 in 2014 (Tremblay et al., 2014), and 37 in 2016 (Tremblay et al. 2016b). The consolidated findings from participating countries were summarized in the form of a global matrix of grades from 15 countries in 2014 and from 38 countries in 2016.

Results from the Global Matrix 1.o (Tremblay et al., 2014) showed a significant global variation in children's sedentary behaviours, although sedentary behaviour through screen time was found to be too high in the majority of countries where it was assessed (average of D). Similar results were obtained in the second edition of the Global Matrix (Tremblay et al., 2016b).

In addition, Roman-Vilas et al.(2016) evaluated adherence to new 24-hour movement guidelines (CSEP, 2016) among 6,128 children aged 9 to 11 years, across 12 countries participating in the International Study of Childhood Obesity, Lifestyle and the Environment (ISCOLE). They found that only $39.3 \%$ of children from all study sites met the screen time duration recommendations (no more than 2 hours per day).

These results show that childhood engagement in sedentary behaviours is too high in the majority of countries where it has been assessed, highlighting the need for interventions that reduce sedentary time among the pediatric population.

\section{Determinants of sedentary behaviours among children and youth}

According to Sallis et al. (2008), multiple determinants across multiple levels and sectors (individual, social, physical environments, and policies) must be addressed to achieve population change in sedentary behaviour. Because this is a newer area of health research, it still lacks a conceptual framework incorporating the potential determinants of children's sedentary behaviours at different levels (e.g., policy environment, behaviour settings, individual factors). Such a framework could further help illustrate the complexity of the sedentary behaviour phenomenon among children and youth, guide research, and support the development of interventions and policies.

Research on determinants of sedentary behaviours is still at an early stage, and much of the available evidence is in relation to TV viewing time. However, an overview of the available findings follows.

\subsection{The Ecologic Model of Sedentary Behaviours}

To aid research on factors that influence sedentary behaviours, an ecologic model of four domains of sedentary behaviour (Figure 3) was developed by Owen et al. in 2011. The main objective of this model is to focus attention on the domains within which the relevant contextual factors such as environmental, social, or organizational, influence sedentary behaviours and interact with individual-level attributes (e.g., preferences, enjoyment or barriers) and social factors (e.g., family demands or workplace relationships).

According to Owen et al. (2011), this model covers factors relevant to most age groups; many influences are likely to operate similarly for children, youth, adults, and older adults, but other influences will be distinct for these different age groups. For example, school-based initiatives such as reducing sitting in physical education time will particularly affect children. This ecological model has the strength to propose an adequately complex summary of potential sedentary behaviour factors of influence and is a useful tool for the development of strategies to reduce sedentary behaviours. However, further development of the model is warranted to incorporate the characteristics and consequences on health of the sedentary behaviour phenomenon.

\subsection{Mapping the environmental and social contexts of sed- entary behaviour and health}

A narrative review mapping the environmental and social contexts of sedentary behaviour and health was published by Owen et al. in 2014. The authors proposed a conceptual model showing relationships that needed to be identified in the study of sitting behaviours (Figure 4). The authors aimed to provide a basis for new research perspectives on 


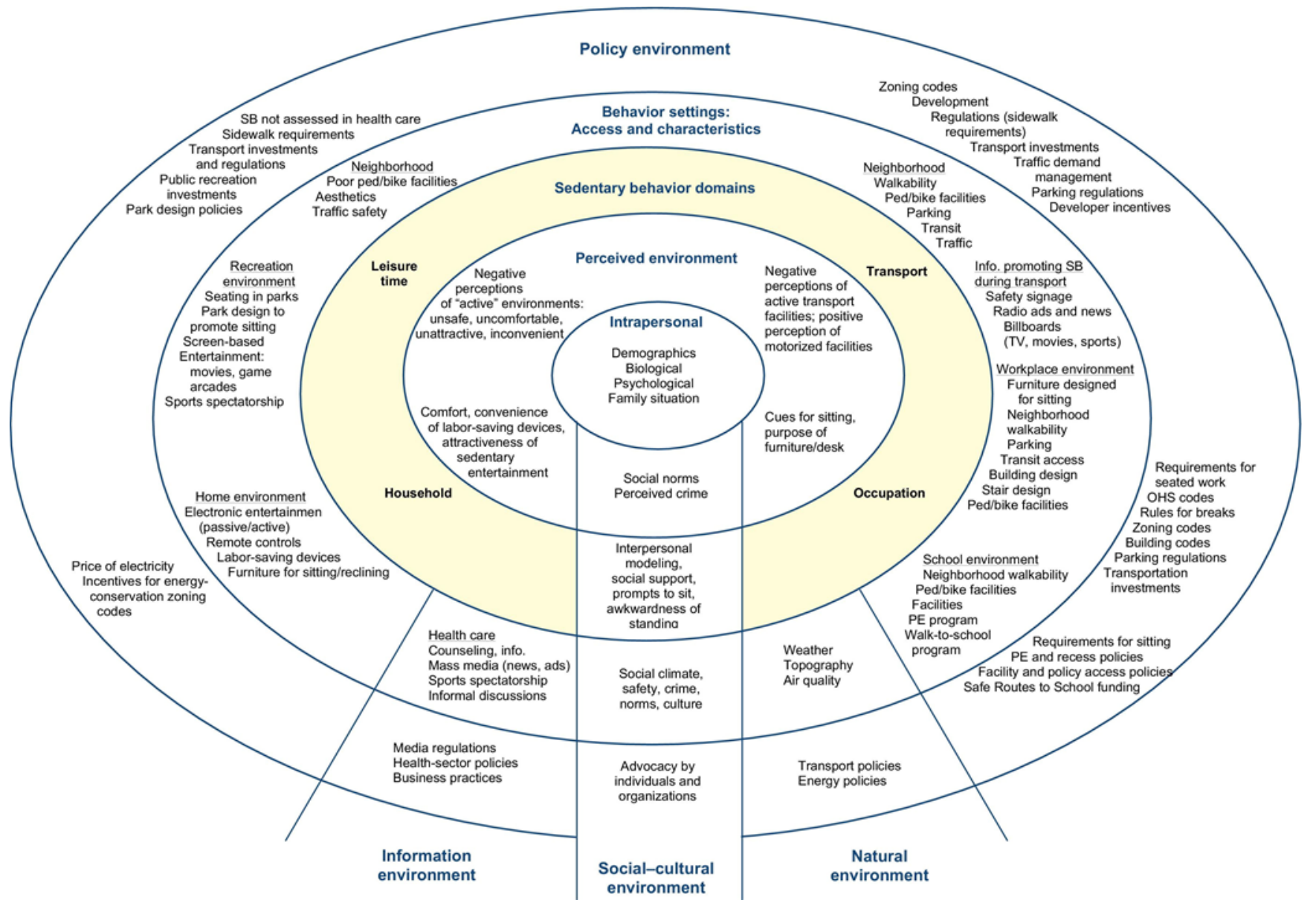

Ecologic model of four domains of sedentary behaviour: OHS, occupational health and safety; PE, physical educa-
Figure 3 ion, pedestrian; SB, sedentary behaviour. Reprinted from "Adults' Sedentary Behaviour: Determinants and
Interventions," by N. Owen, T. Sugiyama, E. E. Eakin, P. A. Gardiner, M. S. Tremblay, and J. F. Sallis, 2011, Ameri-
can Journal of Preventive Medicine, 41, 191. Copyright 2011 by American Journal of Preventive Medicine. Reprinted
with permission.

the adverse health outcomes of sitting time, within a broader understanding of environmental influences on health behaviours, taking an ecological and socioeconomic inequalities perspective across life stages.

This recent model has the advantage of being well organized and gives an interesting approach to the environmental settings of influences, the mediation and moderation factors, the intermediate risk factors, and the major health outcome associated with sitting time. However, this model is not specific to children. In addition, the complexity of sedentary behaviour is not well presented and it is reduced to the most recurrent "sitting time" behaviour settings. Finally, the policy environment level (such as media regulation or public recreation investment) present in the previous ecological model is missing in the "environmental settings influences".

\section{Proposition of a conceptual model of seden- tary behaviour among children and youth}

The strengths of both previously presented models and other elements described herein, have been combined to develop a conceptual model of sedentary behaviour among children and youth (Figure 5). This new model retains the organization of the second model (Owen et al., 2014) but replaces the environmental setting influences by a summary of the determinants from the ecologic model of Owen et al. (2011). In addition, a summary of the determinants to children is presented and updated with a recent systematic review of determinants of sedentary behaviour in youth (Stierlin et al., 2015). Quantitative and qualitative characteristics of sedentary behaviour presented previously in this paper (see section entitled "Sedentary behaviours, complexity of the phenomenon") have been chosen to replace the 
EnvironmentalSettings Influences

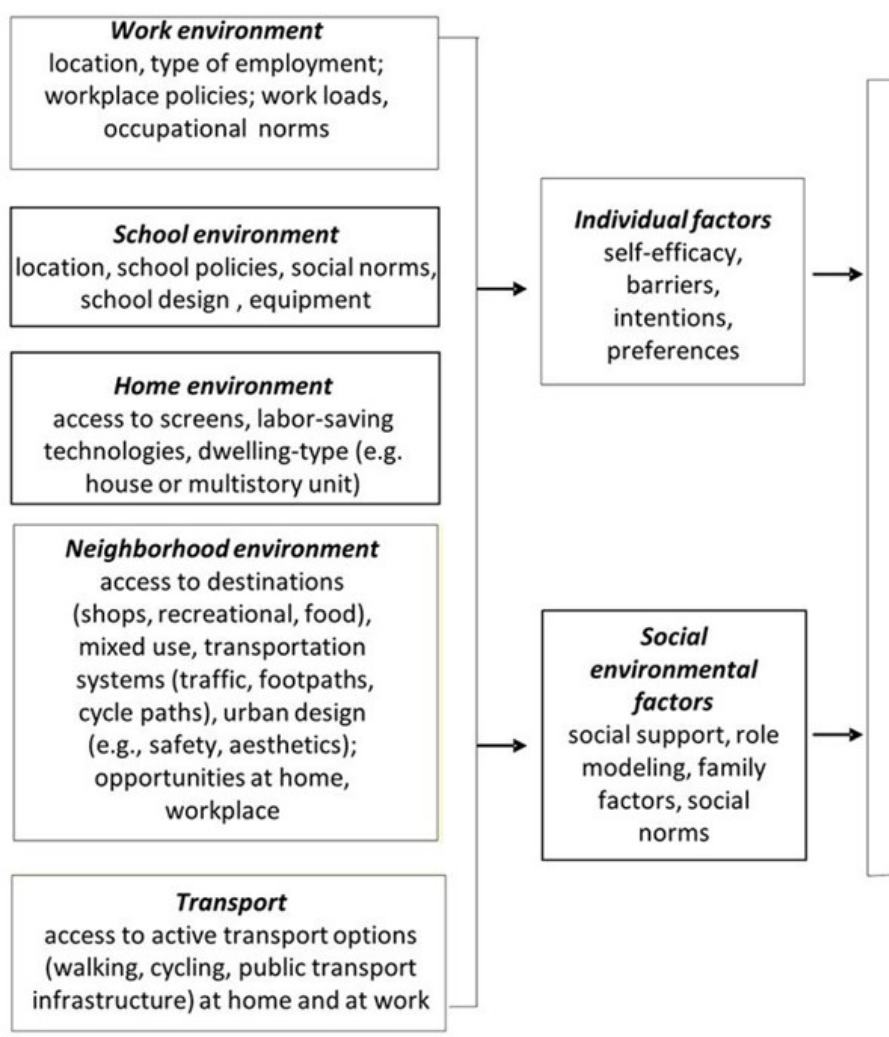

\section{Sitting Time \\ Intermediate Risk Markers Major Health Outcomes}

Moderation (demographic and biological factors age, gender, race/ethnicity, socioeconomic status, adiposity, genetics) across key life stages: childhood, adolescence, adulthood, older adulthood

\section{Overview of relationships that need to be identified - between the built, policy and social environments, prolonged
sitting in particular. Reprinted from "Sedentary Behaviour and Health: Mapping Environmental and Social Con- texts to Underpin Chronic Disease Prevention," by N. Owen, J. Salmon, M. J Koohsari, G. Turrell, and B. Giles- Corti, 2014, British Journal of Sports Medicine, 48, p. 175. Copyright 2014 by BMJ Publishing Group Limited. Re- printed with permission.}

sitting time settings. The "intermediate risk markers" and the "major health outcomes" sections from Owen et al. (2014) model have also been completed with the findings presented previously in the part reviewing the consequences of childhood sedentary behaviours. The individual factors have been presented as determinants, moderators or mediators in this model because it is assumed that their effects will vary in different countries or contexts, where environmental, social, and cultural attributes influencing sedentary behaviour may differ. This "Conceptual model for the study and understanding of children and youth's sedentary behaviour" is not meant to be exhaustive in its different categories but tries to give an overview of the available evidence and potential inferences in the literature.

The proposed model aims to inform broad, evidence-based environmental and policy initiatives. Moreover, it encour- ages further research on sources of influence on specific sedentary behaviours in different contexts and their direct and indirect consequences on health. The links between each of the elements in this model need to be studied in various countries, where environmental, social, and cultural attributes influencing sedentary behaviours may differ (Owen et al., 2011). For example, it would be interesting to assess if a high socio-economic status is associated with decreased sedentary time among children in high or very highincome countries and increased sedentary time among children in low-income countries.

\section{Gaps in the literature}

Because sedentary behaviour related health research is a burgeoning area of study, there are still many gaps in the literature. However, some initiatives are seeking to address these needs. To standardize the methodology used in stud- 


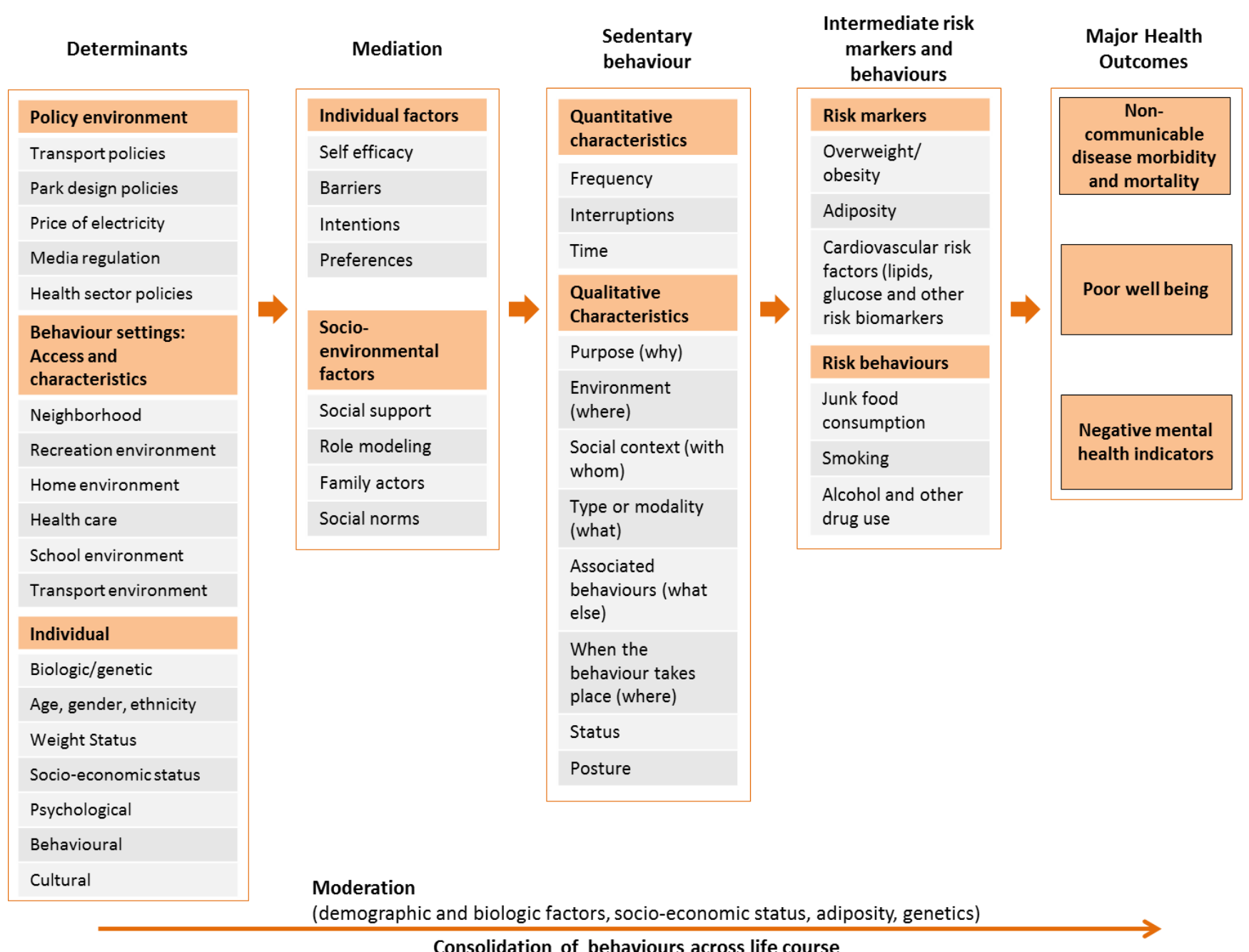

Consolidation of behaviours across life course

Figure 5 Conceptual model for the study and understanding of children and youth's sedentary behaviour.

ies in terms of sedentary behaviour (and physical activity) measurements, the International Society for the Measurement of Physical Behavior was created. It is now organizing an annual congress and sharing resources on its website. In addition, the SIT project, referred to above, is still ongoing.

Evidence on the potential moderating role of socioeconomic inequality on sedentary behaviours and their adverse health outcomes are needed from a multilevel, longitudinal, and life-course perspective (Owen et al., 2014). Future research also needs to examine the common and distinct influences of environmental, social, and other relevant determinants of sitting time in relevant settings (Owen et al., 2014).

It is also necessary to take into consideration the complexity of sedentary behaviours for the development of policies that target sedentary time among children and youth. All types of sedentary behaviours may not be equally deleterious for health. It is of utmost importance to identify the sedentary behaviours associated with the most negative impacts on health, their specific determinants, their mediators and moderators in different countries, while also considering different environmental, social, and cultural contexts.

Further research is also needed to identify the modifiable environmental and social determinants of sedentary behaviour to better understand how to reduce them and work towards population-wide strategies targeting prolonged sitting time (Owen et al., 2014).

Finally, identifying the important correlates at multiple levels for sitting time in different settings is also required. For instance, time spent sitting in cars is associated with urban design and transportation factors while sitting at work or in the domestic environment may be associated with arrangements of furniture, communication technology, and proximal-social factors (Owen et al., 2011). 


\section{Conclusion}

Sedentary behaviours are common and complex behaviours that can potentially affect the health of children and youth on both a physiological and a psychological level, depending on how they are accumulated throughout the day. While childhood engagement in sedentary behaviours has been found to be too high in the majority of countries where it has been assessed, developing research and policies that target the reduction of sedentary behaviours among children and youth must be ranked as a top priority for all public health organizations worldwide. Although further research is needed to identify specific determinants and consequences of different types and patterns of sedentary behaviours in various contexts, the Conceptual Model for the Study and Understanding of Children and Youth's Sedentary Behaviour proposed in this paper, gives an overview of the topic and supports the development of specific actions.

\section{Acknowledgements}

I want to thank Dr. Alejandra Dubois, whose class inspired this work as an original assignment, for proofreading, and editing the work. Thank you to Miss Kathryn LaRoche, Miss Nicole Bergen, Mr. Fabien Rivière and Mr. Philippe Turcotte for providing comments and reviewing drafts of this paper.

\section{References}

Altenburg, T. M., \& Chinapaw, M. J. (2015). Bouts and breaks in children's sedentary time: currently used operational definitions and recommendations for future research. Preventive medicine, 77, 1-3.

Biddle, S. J., Petrolini, I., \& Pearson, N. (2014). Interventions designed to reduce sedentary behaviours in young people: a review of reviews. British Journal of Sports Medicine, 48(3), 182-186.

Brown, B. B. (1968). Delphi process: A methodology used for the elicitation of opinions of experts (No. RAND-P3925). RAND CORP SANTA MONICA CA.

Carson, V., Hunter, S., Kuzik, N., Gray, C. E., Poitras, V. J., Chaput, J. P., ... \& Kho, M. E. (2016). Systematic review of sedentary behaviour and health indicators in school-aged children and youth: an update 1. Applied Physiology, Nutrition, and Metabolism, 41(6), S240-S265.

Carson, V., Kuzik, N., Hunter, S., Wiebe, S.A., Spence, J.C., Friedman, A., Tremblay, M.S., Slater, L.G. and Hinkley, T. (2015). Systematic review of sedentary behavior and cognitive development in early childhood. Preventive medicine, $78,115-122$.
Chastin, S. F. M., Schwarz, U., \& Skelton, D. A. (2013). Development of a consensus taxonomy of sedentary behaviors (SIT): Report of Delphi round 1. PLoS One, 8(12), e82313. doi:10.1371/journal.pone.0082313

Chinapaw, M., Altenburg, T., \& Brug, J. (2015). Sedentary behaviour and health in children-Evaluating the evidence. Preventive Medicine, 70, 1-2. doi:10.1016/ j.ypmed.2014.10.029

CSEP. (2016). 24-Hour Movement Guidelines for Children and Youth. Retrieved from: http://www.csep.ca/en/ guidelines/24-hour-movement-guidelines

Gibbs, B. B., Hergenroeder, A. L., Katzmarzyk, P. T., Lee, I. M., \& Jakicic, J. M. (2014). Definition, measurement, and health risks associated with sedentary behaviour. Medecine \& Science in Sports \& Exercise, 47(6), 1295-1300.

doi:10.1249/MSS.0000000000000517

Healy, G. N., Dunstan, D. W., Salmon, J., Cerin, E., Shaw, J. E., Zimmet, P. Z., \& Owen, N. (2008). Breaks in sedentary time beneficial associations with metabolic risk. Diabetes Care, 31(4), 661-666. doi:10.2337/dco7-2046

Kelder, S. H., Perry, C. L., Klepp, K. I., \& Lytle, L. L. (1994). Longitudinal tracking of adolescent smoking, physical activity, and food choice behaviors. American Journal of Public Health, 84(7), 1121-1126.

Owen, N., Healy, G. N., Matthews, C. E., \& Dunstan, D. W. (2010). Too much sitting: the population-health science of sedentary behavior. Exercise and sport sciences reviews, 38 (3), 105 .

Owen, N., Sugiyama, T., Eakin, E. E., Gardiner, P. A., Tremblay, M. S., \& Sallis, J. F. (2011). Adults' sedentary behaviour: Determinants and interventions. American Journal of Preventive Medicine, 41(2), 189-196. doi:10.1016/

j.amepre.2011.05.013

Owen, N., Salmon, J., Koohsari, M. J., Turrell, G., \& GilesCorti, B. (2014). Sedentary behaviour and health: Mapping environmental and social contexts to underpin chronic disease prevention. British Journal of Sports Medicine, 48(3), 174-177. doi:10.1136/bjsports-2013-093107

ParticipACTION. (2015). The biggest risk is keeping kids indoors. The 2015 ParticipACTION report card on physical activity for children and youth. Toronto: ParticipACTION. Retrieved from: http://www.participaction.com/sites/ default/files/downloads/Participaction-2015ReportCardFullReport_4.pdf

Public Health Agency of Canada. (2013). What is popula- 
tion health? Ottawa: Public Health Agency of Canada. Retrieved from: http://www.phac-aspc.gc.ca/ph-sp/approach -approche/appr-eng.php

Sallis, J. F., Owen, N., \& Fisher, E. B. (2008). Ecological models of health behavior. Health behavior and health education: Theory, research, and practice, 4, 465-486.

Salmon, J., Tremblay, M. S., Marshall, S. J., \& Hume, C. (2011). Health risks, correlates, and interventions to reduce sedentary behaviour in young people. American Journal of Preventive Medicine, 41(2), 197-206. doi:10.1016/ j.amepre.2011.05.001

Saunders, T. J., Chaput, J. P., \& Tremblay, M. S. (2014). Sedentary behaviour as an emerging risk factor for cardiometabolic diseases in children and youth. Canadian Journal of Diabetes, 38(1), 53-61. doi:10.1016/

j.jcjd.2013.08.266

Sedentary Behaviour Research Network. 2012. Letter to the editor: Standardized use of the terms sedentary and sedentary behaviours. Applied Physiology, Nutrition, and Metabolism. 37(3): 540-542. doi:10.1139/h2012- 024

Stierlin, A. S., De Lepeleere, S., Cardon, G., DargentMolina, P., Hoffmann, B., Murphy, M. H., . . .De Craemer, M. (2015). A systematic review of determinants of sedentary behaviour in youth: A DEDIPAC-study. International Journal of Behavioral Nutrition and Physical Activity, 12 (1), 1-19. doi: 10.1186/s12966-015-0291-4

Suchert, V., Hanewinkel, R., \& Isensee, B. (2015). Sedentary behaviour and indicators of mental health in schoolaged children and adolescents: A systematic review. Preventive Medicine, 76, 48-57. doi:10.1016/ j.ypmed.2015.03.026

Tremblay, M. S., Colley, R. C., Saunders, T. J., Healy, G. N., \& Owen, N. (2010). Physiological and health implications of a sedentary lifestyle. Applied Physiology, Nutrition, and Metabolism, 35(6), 725-740. doi: 10.1139/H10-079

Tremblay, M. S., LeBlanc, A. G., Kho, M. E., Saunders, T. J., Larouche, R., Colley, R. C., . . Gorber, S. C. (2011). Systematic review of sedentary behaviour and health indicators in school-aged children and youth. International Journal of Behavioral Nutrition and Physical Activity, 8 (1), 98.

Tremblay, M. S., LeBlanc, A. G., Carson, V., Choquette, L., Connor Gorber, S., Dillman, C., .. .Kho, M. E. (2012). Canadian sedentary behaviour guidelines for the early years (aged 0-4 years). Applied Physiology, Nutrition, and Me- tabolism, 37(2), 370-380.

Tremblay, M. S., Gray, C. E., Akinroye, K., Harrington, D. M., Katzmarzyk, P. T., Lambert, E. V., .. .Prista, A. (2014). Physical activity of children: a global matrix of grades comparing 15 countries. Journal of physical activity and health, 11(s1), S113-S125.

Tremblay, M. S., Carson, V., \& Chaput, J. P. (2016a). Introduction to the Canadian 24-Hour Movement Guidelines for Children and Youth: An Integration of Physical Activity, Sedentary Behaviour, and Sleep 1. Applied Physiology, Nutrition, and Metabolism, 41(6), iii-iv.

Tremblay, M. S., Barnes, J. D., González, S. A., Katzmarzyk, P. T., Onywera, V. O., Reilly, J. J., \& Tomkinson, G. R. (2016b). Global Matrix 2.0: report card grades on the physical activity of children and youth comparing 38 countries. Journal of physical activity and health, 13(11 Suppl 2), S343-S366.

Tremblay, M. S., Aubert, S., Barnes, J. D., Saunders, T. J., Carson, V., Latimer-Cheung, A. E., . . Chinapaw, M. J. (2017). Sedentary Behavior Research Network (SBRN)Terminology Consensus Project process and outcome. International Journal of Behavioral Nutrition and Physical Activity, 14(1), 75. 